\title{
SMART FARMING: ORGANIC COMMUNICATION CHANNELS (OCCS)
}

\author{
Aaron Roopnarine ${ }^{1 *}$ And Sean Rocke ${ }^{2}$ \\ ${ }^{1,2}$ Faculty of Engineering, The University of the West Indies, Trinidad \\ ${ }^{1}$ Email: aaron.roopnarine@sta.uwi.edu*(Corresponding author) \\ 2Email: sean.rocke@sta.uwi.edu
}

\begin{abstract}
Organic Communication Channels (OCCs) are any hydrocarbon-based media which can be used to communicate data. This has great potential when applied to cyberphysical systems such as with smart farming. This paper establishes the relevance (though a literature survey and consideration of possible use cases for this technology), feasibility (through channel characterisation experiments) and proposes the infrastructure for OCC systems. The results show feasible communication of voice, video and text is possible with the proposed infrastructure for OCC communication. Finally, the approach of the proposal is evaluated through considerations for future work.
\end{abstract}

Keywords: Human Body Communication (HBC), Internet Of Farming (Iof), Organic Communication Channels (OCC), Path Loss, Propagation Modelling, Smart Farming.

https://doi.org/10.47412/FUAP1192

\section{Introduction}

Organic Communication Channels (OCCs) are any hydrocarbon-based media used to communicate information. These include, but are not limited to, humans, plants, animals and soil. The accomplishments of Human Body Communication (HBC) — which uses the human body as the media to communicate datafor medical telemetry in Body Area Networks (BANs) prove that there is potential with OCCs as an alternative to short range Radio Frequency (RF) communication as it facilitates more secured communication, higher data rates, better spectral efficiency and greater power efficiency [1], [2]. However, to the extents of the papers surveyed, not much work has been done to extend the HBC techniques to the other OCC scenarios. Soil-based communication has yielded relatively poor data rates [3], [4] with no internationally agreed upon form of standardised communication.

Smart Farming is the application of IoT (Internet of Things) to agriculture. OCCs show promise when applied to smart farming use cases as illustrated in Figure 1. Thus, OCCs have great potential when considering low power communication and channel sensing. However, smart farming currently does not employ OCCs. RF-based technologies - e.g. 3G technology, Bluetooth, ANT+, WiFi and LoRA - are typically used for telemetry [5] - [9]. If OCC transceivers are implemented such that OCCs supplement RF communication techniques, as shown in Figure 1, there will be an amelioration of data reliance. Figure 1 also shows channel sensing can be employed to acquire sensor data- which include but is not limited to plant growth state, fruit quality, soil moisture content and animal activity. 
Figure 2 shows a specific use case of an organic area network (OAN). OANs can be integrated into smart farming applications. OANs can be used in applications such as soil moisture regulation as proposed by [9] as opposed to utilizing $3 \mathrm{G} / 4 \mathrm{G}$ networks. This offers the option to simultaneously transmit and measure soil parameters through integration of channel sensing technologies. Additionally, OCC transceivers improve data resilience when used to supplement RF-based communication techniques.

In [5] the authors characterized channels between animals in the $868 \mathrm{MHz}$ band. However, channel characterization within organic objects is less studied. Extensive work has been done regarding humanbased OCC channel characterization [1], [10]-[16]. From the extents of the literature survey, no work has been done regarding vegetation-based OCC characterization. Soil-based OCCs have been characterized for magnetic-induction as the communication technique but there is no standardized form of communication soil-based OCCs and across other OCCs [17] - [20]. The evaluation of OCC performance and feasibility is dependent of channel characterization. Thus, to address these research gaps, this paper characterizes vegetation-based and soil-based organic channels in addition to proposing a standardized framework for OCC communication based on HBC techniques.

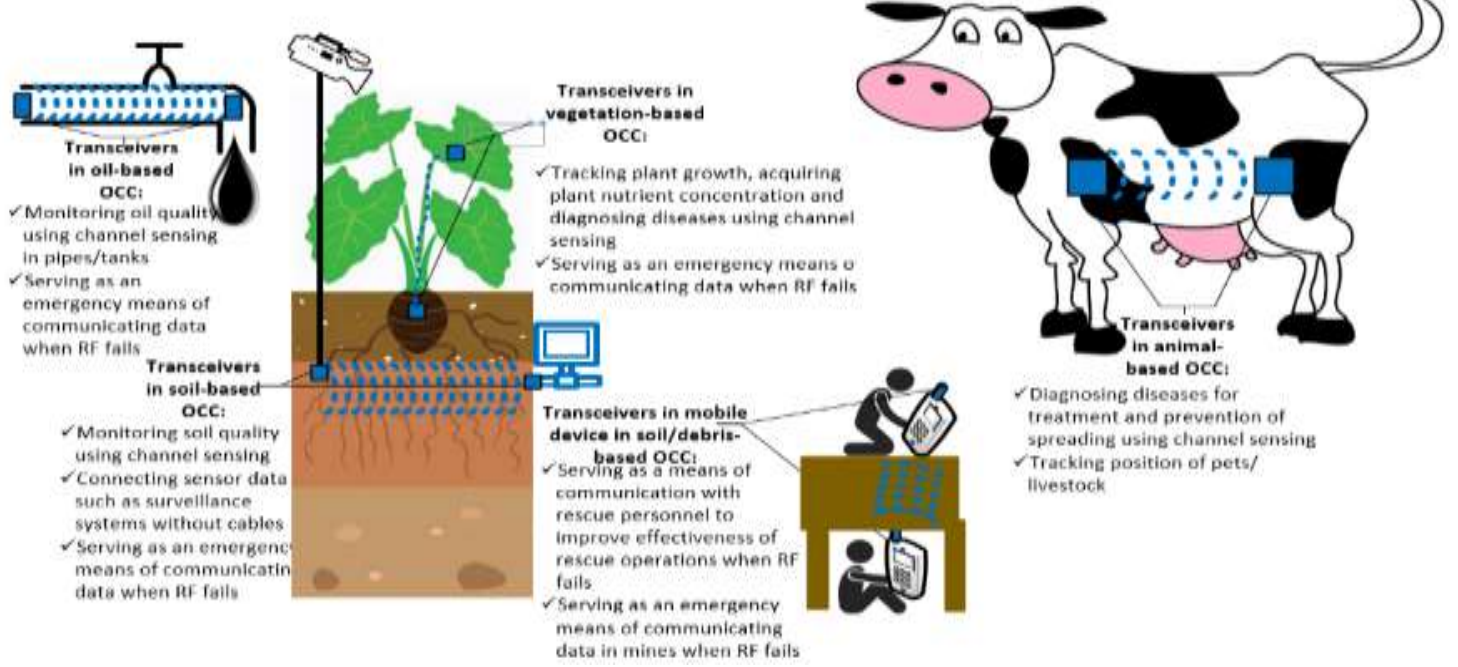

Figure 1: Use cases for OCCs in smart farming scenarios

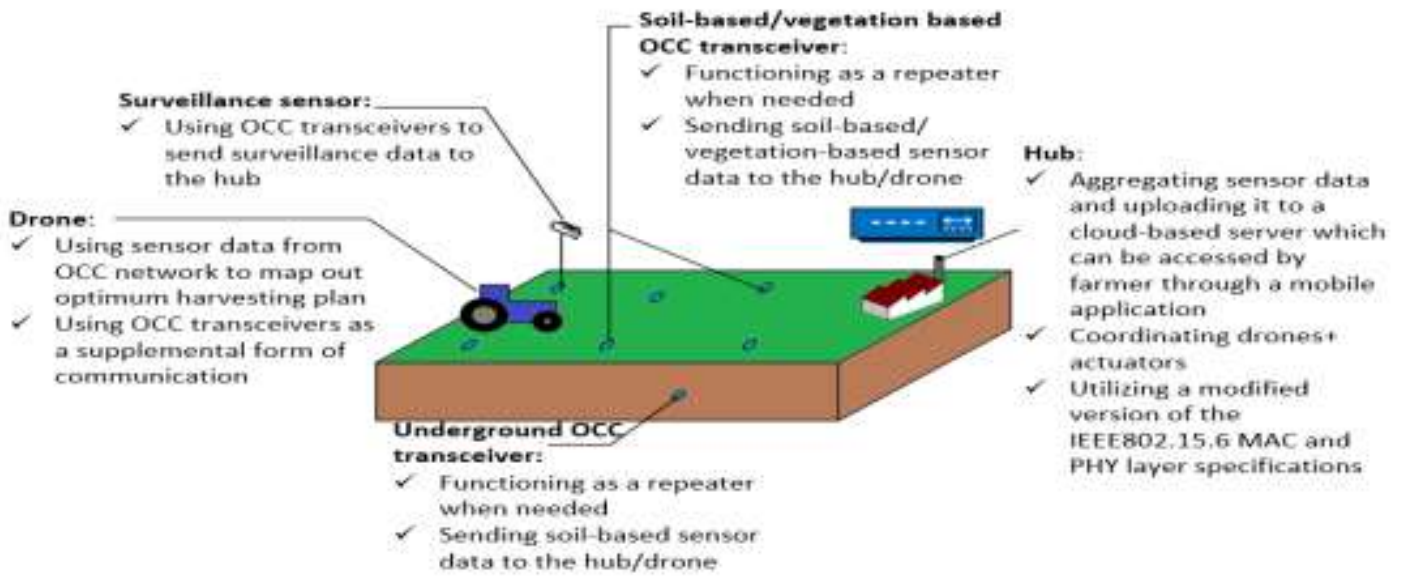

Figure 2: A specific use case of an organic area network (OAN) 


\section{Methodology}

This section covers how the objectives of this research is achieved through channel characterization, OCC framework proposal

\subsection{Channel Characterization}

The OCCs channel characterization followed the stages: electrode design, channel measurements and derivation of propagation model (in this case, pathloss equations).

The OCCs investigated were a papaya stem (to represent herbaceous vegetation-based OCCs), a coconut stem (to represent woody vegetation-based OCCs) and a plastic trough filled with loam soil with chive plants growing (to emulate roots in soil-based OCCs).

Figure 3(a) shows the electrode designed for OCC communication. HBC techniques can be categorized as either eHBC (electric HBC) — which utilize electric fields through capacitive coupling and galvanic coupling methods - or mHBC (magnetic HBC) - which utilize magnetoquasistatic fields through magnetic induction [1], [21]. These were considered in the electrode design for OCC channel characterization measurements. mHBC allows for channel resilience since the magnetic permeability of most materials are the same [10]. eHBC is standardized through the IEEE 802.15.6 standard [22] and allows for a greater range of frequency operation through the capacitively coupled technique. Consequently, the $60 \mathrm{~cm} \times 60 \mathrm{~cm}$ FR 4 PCB (Printed circuit board) with spiralled copper geometry, in Figure 3(a), was chosen. Thus, the modulated signal will propagate through the OCC by means of both electric fields - through capacitive and galvanic coupling - and magnetoquasistatic fields - through magnetic induction.

Figure 3(b) shows the general experimental setup for channel characterization. The Vector Network Analyser (VNA) sends a known signal to the OCC through the electrodes from the Device Under Test (DUT) port. This signal passes through the organic channel and is collected at the receive electrodes and sent to the DET port of the VNA where the effect on the magnitude and phase of the signal inputted are calculated. This makes it possible to calculate the $S_{21}$ parameter or transmission coefficient through Eq. (1).

$$
S_{21}=20 \log _{10}\left(\frac{V_{R x}}{V_{T x}}\right)
$$

Where: $V_{T X}$ is the voltage of the signal sent from the DUT port and $V_{R x}$ is the voltage of the signal received at the Detection (DET) port. Therefore, the frequency response of the OCC investigated can be derived. Baluns decouple the transmitter (DUT port) and receiver (DET port). In a typical sensor use case, transceiver grounds are not electrically connected to the same potential via a perfect conductor. Baluns address this by isolating these grounds. Consequently, the effect of the grounds of the function generator and spectrum analyser is removed. The Baluns' effect on the channel response was factored into the measurement and calibration. 


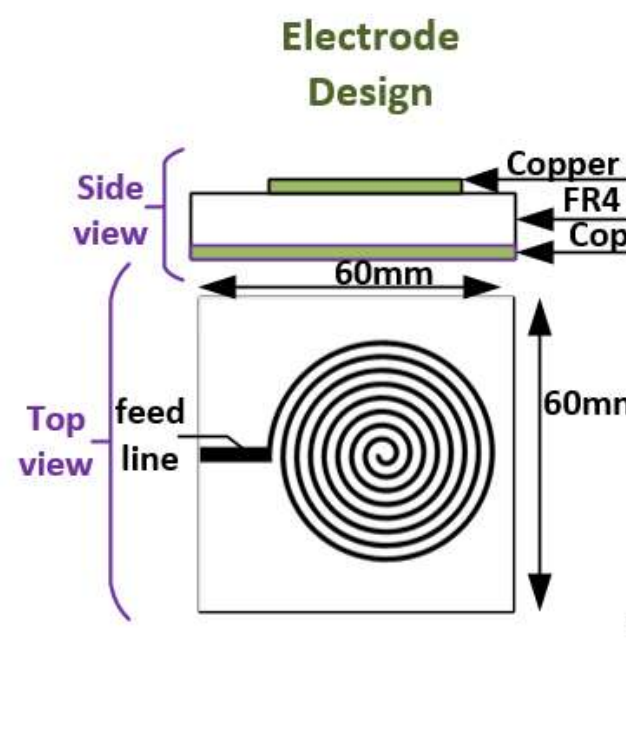

(a)
Experimental

set-up

VNA

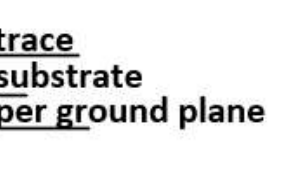

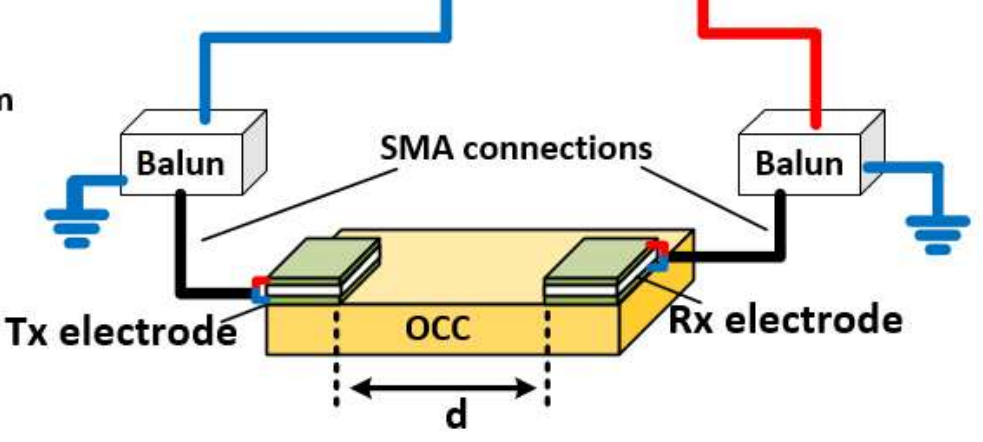

(b)

Figure 3: (a) The OCC electrode design (b) The general set-up for OCC Channel Characterization

There has already been considerable work done in the HBC band $(18.375 \mathrm{MHz}-23.625 \mathrm{MHz})$ as specified by the IEEE 802.15 .6 standard. Therefore, channel characterization was done over the HBC band to assess the feasibility of utilizing HBC standards for non-HBC OCC use cases.

The following were the steps taken for channel characterization:

1. Setting up the equipment as shown in Figure 3(b) for $42.5 \mathrm{~cm}$ (maximum channel length) of coconut stem as the OCC. The PCB electrodes were placed such that the copper coated spiral was in contact with the organic channel. The channel length, $d$, was initially set to $2.5 \mathrm{~cm}$

2. Calibration and configuration of the VNA for transmission mode operation in the HBC band. The transmission power was set to $1 \mathrm{~mW}$ in accordance with the ICNIRP standard for general exposure of humans to electric and magnetic fields [23]. Plants may accept more power, but with the goal of universal OCC standard, minimizing human exposure takes precedence [24].

3. Collection of $S_{21}$ parameters. The VNA was configured to send the input signals and the magnitude and phase of the $S_{21}$ parameter was noted. The above steps were repeated varying $d$ until it became equal to the maximum channel length.

4. Repeating steps 1-3 for
a. $\quad 37.5 \mathrm{~cm}$ of papaya stem and
b. $37.5 \mathrm{~cm}$ of loam soil

\section{Proposed Scheme}

Adopting a standardised form of communication for all OCCs improves spectral efficiency. HBC has been standardised through the IEEE 802.15.6 standard for safe communication using eHBC techniques. Thus, this standard can be adopted for other OCCs. Relatively high resonance frequencies exist for soil-based OCCs in the HBC band [25]. This lends credence to applying the IEEE802.15.6 standard to soil-based OCCs. 
Table 1 shows the proposed changes to the HBC PHY (Physical layer) of the IEEE 802.15.6 standard for standardized OCC PHY layer communication.

Table 1: The proposed changes to the IEEE 802.15.16 standard for standardized OCC communication

\begin{tabular}{|c|c|}
\hline Change Implemented & Reason \\
\hline $\begin{array}{l}\text { Gold code generator should not be implemented } \\
\text { to generate the preamble and start frame delimiter } \\
\text { (SFD) generation. The output of the gold code } \\
\text { generator should be used instead since it is the } \\
\text { same for all cases }\end{array}$ & \multirow[t]{2}{*}{$\begin{array}{l}\text { To reduce transceiver complexity and hence } \\
\text { improve transceiver power efficiency }\end{array}$} \\
\hline $\begin{array}{l}\text { Serial to parallel conversion should not be } \\
\text { implemented. The output of this computational } \\
\text { block is the same as it's input }\end{array}$ & \\
\hline $\begin{array}{l}\text { CRC8 calculation calculates CRC8 bits for any } \\
\text { polynomial }\end{array}$ & For upgradability \\
\hline $\begin{array}{l}\text { The maximum PSDU (physical layer service data } \\
\text { unit) length should be assumed to be } 255 \text { bytes to } \\
\text { correspond to the input to the Scrambler block } \\
\text { from the MAC (Medium Access Control) layer }\end{array}$ & $\begin{array}{l}\text { To address irrationalities. There is a section in the } \\
\text { standard that implies the PSDU length consists of } \\
\text { the pilot sequence along with the spread frame } \\
\text { which limits to range of the MAC frame to an } \\
\text { impracticable length. }\end{array}$ \\
\hline Pilot sequence not included at the end of frame & $\begin{array}{l}\text { The IEEE } 802.15 .6 \text { standard consists of a valid } \\
\text { example that contradicts the stated requirement } \\
\text { for pilot insertion at the end of the frame. An } \\
\text { assumption was made that there is no pilot } \\
\text { insertion at the end of the frame since it is } \\
\text { reflected in a framing example given in the same } \\
\text { standard. Examples are generally } \\
\text { intended to clarify an initial statement. }\end{array}$ \\
\hline
\end{tabular}

The OCC PHY proposed was simulated with the channel models derived in section 2.1 in MATLAB to evaluate feasibility of communicating.

\section{Results and Analysis}

The log-distance fading model is adopted as the form of channel model representation since it is widely used to model similar communication scenarios. For example, the CM3 model is a log-distance fading model adopted for human-based OCCs where the electrodes are placed on the body's surface [12]. The CM3 model was developed for devices that comply with the IEEE 802.15.6 standard but path loss equations for the HBC band were not developed. Consequently, the log-distance linear model was chosen to fit path loss for the different OCCs under investigation as shown in Eq. (2). The path loss equation parameters specific to the OCC scenarios under investigation, derived from the empirical results, are found in Table 2.

Where

$$
P L=m \log \left(\frac{d}{d_{0}}\right)+c
$$

- $\quad P L$ is the path loss

- $\quad m$ and $c$ are coefficients for linear fitting for ordinary least square regression

- $d$ is the channel length i.e. Tx-Rx distance in $\mathrm{m}$

- $d_{0}$ is the reference channel length assumed to be $1 \mathrm{~m}$

Table 2: The parameters for the path loss equations from channel measurements 
Faculty of Engineering, The UWI, St. Augustine | June $1^{\text {st }}-5^{\text {th }}, 2020$

\begin{tabular}{|l|l|l|l|l|}
\hline Channel & Frequency & \multicolumn{1}{c|}{$\boldsymbol{m}$} & \multicolumn{1}{c|}{$\boldsymbol{c}$} & $\boldsymbol{R}^{\mathbf{2}}$ \\
\hline Coconut & $18.375 \mathrm{MHz}$ & -11.69 & -77.1426 & 0.49 \\
\cline { 2 - 5 } & $21 \mathrm{MHz}$ & -9.692 & -69.262 & 0.62 \\
\cline { 2 - 5 } & $23.625 \mathrm{MHz}$ & -10.7056 & -68.3823 & 0.56 \\
\hline \multirow{5}{*}{ Papaya } & $18.375 \mathrm{MHz}$ & -11.9155 & -64.163 & 0.73 \\
\cline { 2 - 5 } & $21 \mathrm{MHz}$ & -11.4667 & -59.7738 & 0.75 \\
\cline { 2 - 5 } & $23.625 \mathrm{MHz}$ & -10.9021 & -57.6611 & 0.72 \\
\hline Soil & $18.375 \mathrm{MHz}$ & -5.5908 & -86.4998 & 0.19 \\
\cline { 2 - 5 } & $21 \mathrm{MHz}$ & -9.3167 & -78.9049 & 0.56 \\
\cline { 2 - 5 } & $23.625 \mathrm{MHz}$ & -8.8877 & -76.6951 & 0.68 \\
\hline
\end{tabular}

Figure 4 shows the path loss as transmission frequency varies for different OCC channel lengths. There is approximately a $10 \mathrm{~dB}$ channel loss over the frequencies in the $\mathrm{HBC}$ band for vegetation-based channels i.e. papaya and coconut stems. Thus, vegetation-based OCCs' frequency responses can be approximated to flat fading for the HBC band under the channel lengths investigated. Soil-based OCCs over short channel lengths exhibited relatively flat fading over the same frequency range although having relatively higher channel losses when compared to the other OCCs. However, soil-based OCCs over longer channel lengths displayed significant variation in path loss over the frequency range investigated.

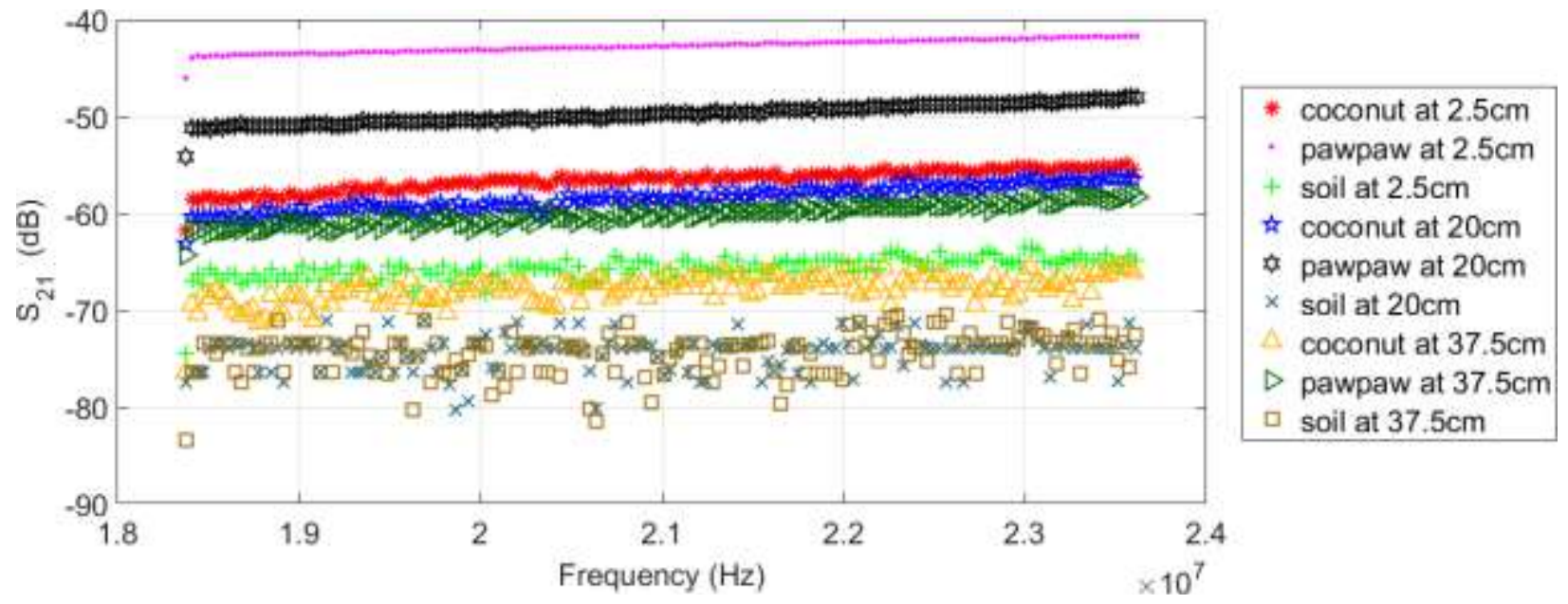

Figure 4: Path loss versus frequency at different distances for OCCs

Figure 5 shows the log-distance path loss over the HBC band. The regression fitting parameters for the empirically collected path losses to the log-distance path loss model is seen in Table 2 . The $R^{2}$ values were low in several instances where fading occurred. Fading becomes apparent as channel length increases. Furthermore, channel losses are significant even at small channel lengths. 


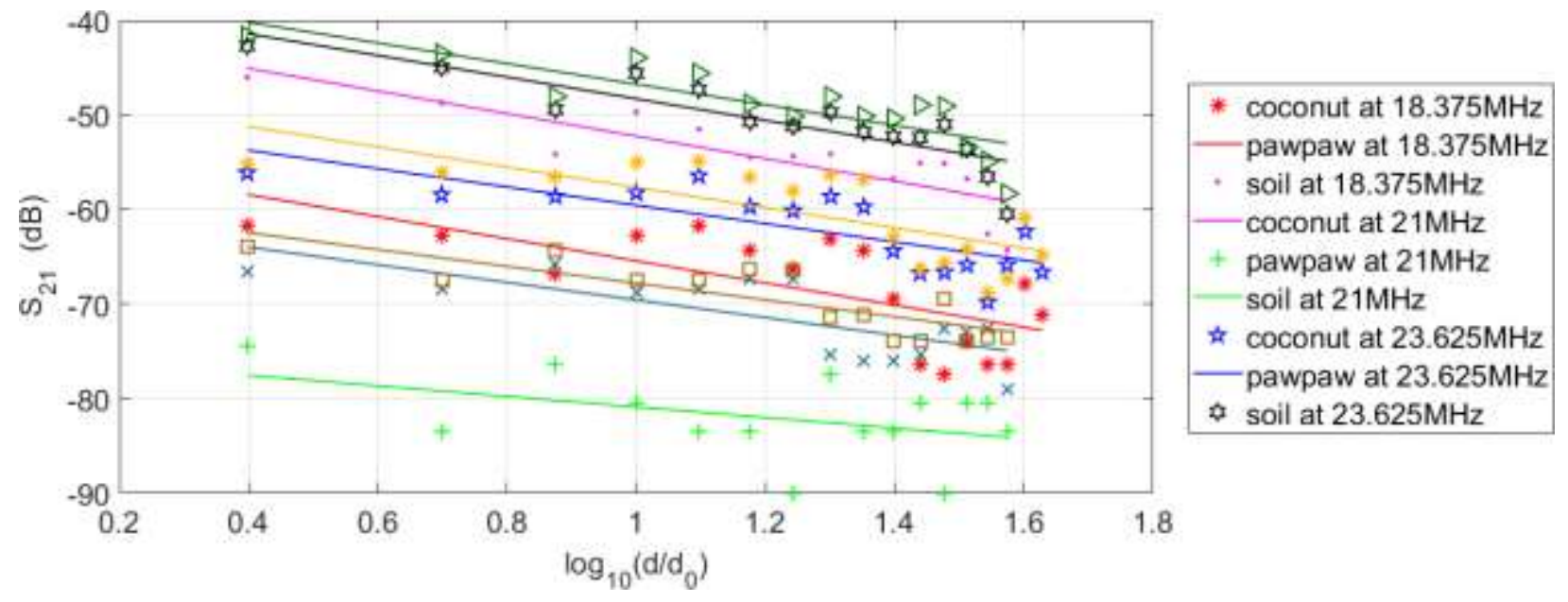

Figure 5: Path loss versus log-distance at different frequencies for OCCs, where $\mathrm{d}=$ channel length $(\mathrm{m})$ and $\boldsymbol{d}_{\mathbf{0}}=1 \mathrm{~m}$

Alternate signal paths between the transmitter and receiver can cause the fading observed. This has been corroborated with research surveyed investigating human-based OCCs. [1] showed the median path loss of roughly $-65 \mathrm{~dB}$ for $30 \mathrm{~cm}$ channel lengths in the same frequency range investigated for channels where capacitive coupling was used as the communication technique. Further, [1] showed frequency selectivity for similar transmission conditions. Hence, the empirical results collected for vegetation-based and soilbased OCCs are comparable with those for human-based OCCs. These similar channel losses lend credence to the feasibility of the use of vegetation-based and soil-based OCCs for intelligible communication with the similar OCC PHY layer specification as that for the IEEE802.15.6 standard.

Figure 6 shows the error rate performance for the OCC PHY layer specification proposed using the channel models derived. Since the system can theoretically achieve a Bit Error Rate (BER) of 0 at data rates greater than $1 \mathrm{kbps}$ in some OCCs, then text data could be transmitted. Since the data rates achieved are above $80 \mathrm{kbps}$ and BERs less than $1 \times 10^{-2}$ can be achieved, this transceiver could be used in for voice transmission at G.711 quality. Since data rates greater than 800kbps can be achieved with BERs less than $1 \times 10^{-2}$, low quality video can be transmitted [26]. 


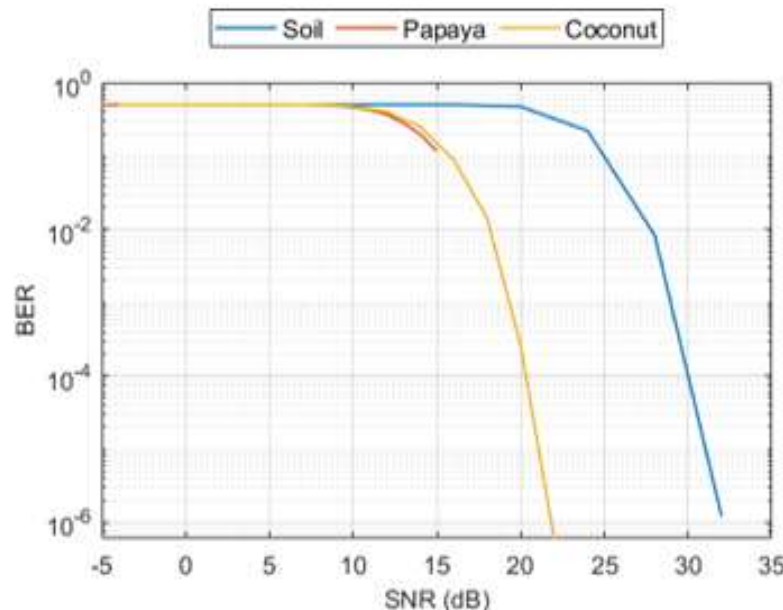

(a)

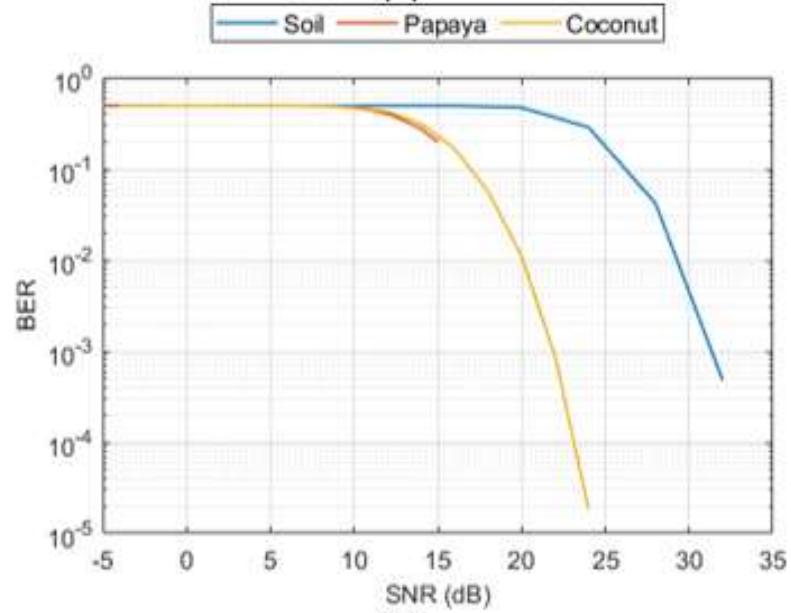

(b)

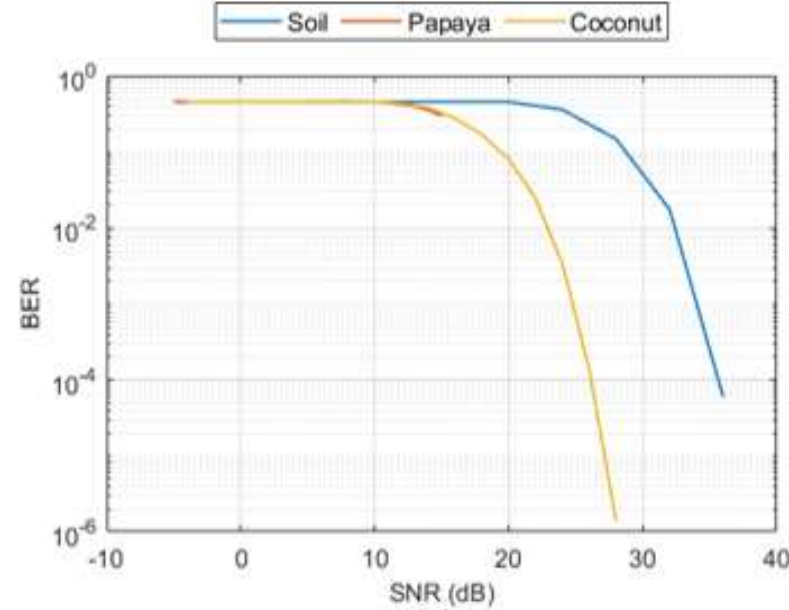

(c)

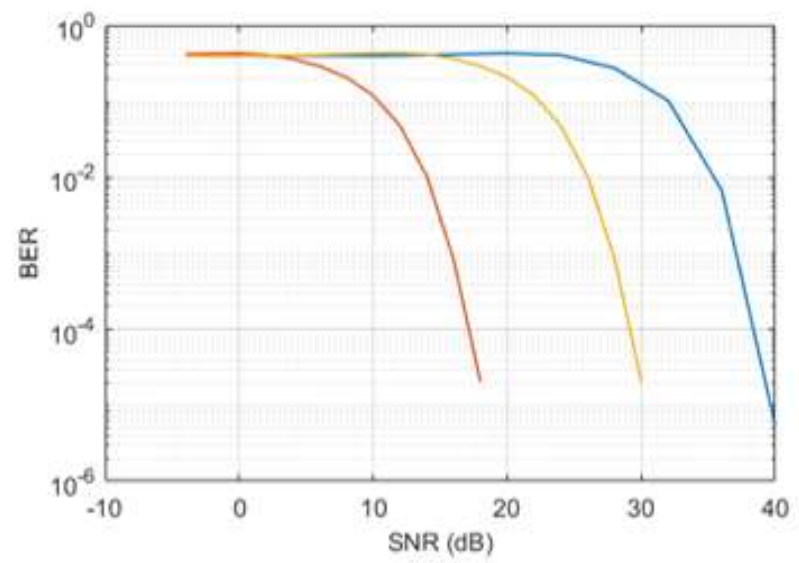

(d)

Figure 6: The BER curves - BER vs Signal to Noise Ratio (SNR) - for the different OCCs investigated using the OCC PHY specification for transmission rates (a) $164 \mathrm{kbps}$ (b)328 kbps (c) $656 \mathrm{kbps}$ and (d)1.3125 Mbps

\section{Conclusion}

OCCs offer great potential when integrated with cyber-physical systems as seen with the examination of use cases for smart farming applications. The performance of systems utilizing OCCs is dependent on the channel characteristics. Vegetation-based (herbaceous and woody) and soil-based OCCs were characterised. The log-distance linear model was used to attempt to fit the magnitude response for the OCCs investigated. The magnitude of the path losses suggest OCCs may be well suited as an alternative to shortrange RF communication as long as there is an organic channel between transceivers. The proposed OCC PHY layer showed feasible communication is possible using OCCs. In further work, dynamic channel models should be investigated for more channel types. Further, the MAC (Medium Access Control) layer for the IEEE 802.15.6 standard should be modified for all OCCs. 


\section{References}

[1] M. D. Pereira, G. A. Alvarez-Botero, and F. R. de Sousa, "Characterization and modeling of the capacitive hbc channel," IEEE Transactions on Instrumentation and Measurement, vol. 64, no. 10, pp. 2626-2635, 2015.

[2] D. Kurup, W. Joseph, E. Tanghe, G. Vermeeren, and L. Martens, "Extraction of antenna gain from path loss model for in-body communication," Electronics letters, vol. 47, no. 23, pp. 1262-1263, 2011.

[3] X. Tan, Z. Sun, and I. F. Akyildiz, "Wireless underground sensor networks: Mi-based communication systems for underground applications." IEEE Antennas and Propagation Magazine, vol. 57, no. 4, pp. 74$87,2015$.

[4] A. R. Silva and M. Moghaddam, "Design and implementation of lowpower and mid-range magneticinduction-based wireless underground sensor networks," IEEE Transactions on Instrumentation and Measurement, vol. 65, no. 4, pp. 821-835, 2016.

[5] S. Benaissa, D. Plets, E. Tanghe, J. Trogh, L. Martens, L. Vandaele, L. Verloock, F. Tuyttens, B. Sonck, and W. Joseph, "Internet of animals: Characterisation of lora sub-ghz off-body wireless channel in dairy barns," Electronics Letters, vol. 53, no. 18, pp. 1281-1283, 2017.

[6] S. Koompairojn, C. Puitrakul, N. Riyagoon, and S. Ruengittinun, "Smart tag tracking for livestock farming," in 2017 10th International Conference on Ubi-media Computing and Workshops (Ubi-Media). IEEE, 2017, pp. 1-4.

[7] C. Kulatunga, L. Shalloo, W. Donnelly, E. Robson, and S. Ivanov, "Opportunistic wireless networking for smart dairy farming," IT Professional, vol. 19, no. 2, pp. 16-23, 2017.

[8] M. H. Memon, W. Kumar, A. Memon, B. S. Chowdhry, M. Aamir, and P. Kumar, "Internet of things (iot) enabled smart animal farm," in 2016 3rd International Conference on Computing for Sustainable Global Development (INDIACom). IEEE, 2016, pp. 2067-2072.

[9] V. V. h. Ram, H. Vishal, S. Dhanalakshmi, and P. M. Vidya, "Regulation of water in agriculture field using internet of things," in 2015 IEEE Technological Innovation in ICT for Agriculture and Rural Development (TIAR), July 2015, pp. 112-115.

[10] T. Ogasawara, A.-i. Sasaki, K. Fujii, and H. Morimura, "Human body communication based on magnetic coupling," IEEE Transactions on Antennas and Propagation, vol. 62, no. 2, pp. 804-813, 2014.

[11] M. H. Seyedi and D. Lai, A Novel Intrabody Communication Transceiver for Biomedical Applications. Springer, 2017.

[12] IEEE Standards Association and others, "IEEE P802.15 Working Group for Wireless Personal Area Networks (WPANs): Channel Model for Body Area Network (BAN)," IEEE, Report IEEE P802.15-080033-040006, 2008.

[13] J. F. Zhao, X. M. Chen, B. D. Liang, and Q. X. Chen, "A review on human body communication: Signal propagation model, communication performance, and experimental issues," Wireless Communications and Mobile Computing, vol. 2017, p. 15, 2017. [Online]. Available:

https://doi.org/10.1155/2017/5842310

[14] N. Zedong, M. Jingjing, C. Hong, and W. Lei, "Statistical characterization of the dynamic human body communication channel at 45mhz," in Engineering in Medicine and Biology Society (EMBC), 2013 35th Annual International Conference of the IEEE. IEEE, 2013, pp. 1206- 1209.

[15] Y. Zhang, B. Kou, D. Fan, Y. Liu, Z. He, and X. Chen, "A dynamic pilot interval adjustment scheme for hbc channel estimation," in Communications in China (ICCC Workshops), 2016 IEEE/CIC International Conference on. IEEE, 2016, pp. 1-5.

[16] T. Aoyagi, M. Kim, J.-i. Takada, K. Hamaguchi, R. Kohno et al., "Body motion and channel response of dynamic body area channel," in 
Antennas and Propagation (EUCAP), Proceedings of the 5th European Conference on. IEEE, 2011, pp. 3138-3142.

[17] T. E. Abrudan, O. Kypris, N. Trigoni, and A. Markham, "Impact of rocks and minerals on underground magneto-inductive communication and localization," IEEE Access, vol. 4, pp. 3999-4010, 2016.

[18] S. Kisseleff, I. F. Akyildiz, and W. Gerstacker, "Interference polarization in magnetic induction based wireless underground sensor networks," in 2013 IEEE 24th International Symposium on Personal, Indoor and Mobile Radio Communications (PIMRC Workshops), Sep. 2013, pp. 71- 75.

[19] J. R. Wait, "Mutual coupling of loops lying on the ground," Geophysics, vol. 19, no. 2, pp. 290-296, 1954.

[20] J. Hendrickx, B. Borchers, D. Corwin, S. Lesch, A. Hilgendorf, and J. Schlue, "Inversion of soil conductivity profiles from electromagnetic induction measurements," Soil Science Society of America Journal, vol. 66, no. 3, pp. 673-685, 2002.

[21] S. Rocke and J. Persad, "Analysis of magnetically-coupled human body communications," in 2015 COMSOL conference in Boston, 2015.

[22] IEEE, "IEEE Standard for local and metropolitan area networks - Part 15.6: Wireless Body Area networks," IEEE Std 802.15.6-2012, pp. 1-271, Feb 2012.

[23] ICNIRP, "Guidelines for limiting exposure to time-varying electric, magnetic, and electromagnetic fields (up to 300 ghz)," Health Phys, vol. 74, no. 4, pp. 494-522, 1998.

[24] M. E. Maffei, "Magnetic field effects on plant growth, development, and evolution," Frontiers in plant science, vol. 5, p. 445, 2014.

[25] S. Kisseleff, I. F. Akyildiz, and W. H. Gerstacker, "Survey on advances in magnetic induction-based wireless underground sensor networks," IEEE Internet of Things Journal, vol. 5, no. 6, pp. 4843-4856, Dec 2018.

[26] Y. Chen, T. Farley, and N. Ye, "Qos requirements of network applications on the internet," Information Knowledge Systems Management, vol. 4, no. 1, pp. 55-76, 2004.

[27] J. Bae, K. Song, H. Lee, H. Cho, and H.-J. Yoo, "A low-energy crystalless double-fsk sensor node transceiver for wireless body-area network," IEEE Journal of Solid-State Circuits, vol. 47, no. 11, pp. 26782692, 2012.

[28] S. S. Mohan, M. del Mar Hershenson, S. P. Boyd, and T. H. Lee, "Simple accurate expressions for planar spiral inductances," IEEE Journal of solid-state circuits, vol. 34, no. 10, pp. 1419-1424, 1999.

[29] A. Kamilaris, F. Gao, F. X. Prenafeta-Boldu, and M. I. Ali, "Agri-' iot: A semantic framework for internet of things-enabled smart farming applications," in 2016 IEEE 3rd World Forum on Internet of Things (WF-IoT). IEEE, 2016, pp. 442-447. 